\title{
In the jungle of cultural complexity
}

\author{
Charlotte E.H. Wilks \& Kirsten H. Blakey \\ Department of Psychology, University of Stirling, Scotland
}

\section{Introduction}

The jungle - an area overgrown with dense forest and tangled vegetation - is not unlike the concept of complexity within cultural evolutionary research. The notion of 'complexity' has been used across many branches of cultural evolutionary research to describe and explain key concepts, such as cumulative cultural evolution (CCE) and 'complex' cognition. However, whilst interest from diverse research areas has enriched the literature, it has also led to a dense network of inconsistencies which need to be addressed. What are researchers from different areas trying to describe and capture when they refer to complexity? The Culture Conference 2018 aimed to provide a platform in which researchers from different disciplines could further integrate their ideas and begin to untangle their views on the relevance and use of this concept.

The University of Stirling, Scotland, played host to Culture Conference 2018: "Complexity in Culture", following a move from its previous home at the University of Birmingham where it was founded by Elisa Bandini and Dr Eva Reindl. During the two-day conference $\left(12^{\text {th }} \& 13^{\text {th }}\right.$ July) the 63 attendees (Figure 1) were treated to presentations of 18 posters, 11 talks (including four by invited keynote speakers), and an enlightening panel discussion hosted by Dr Anne Kandler. A wide range of disciplines were represented across attendees and speakers. These included comparative, developmental, and experimental psychology; animal behaviour; archaeology; cognitive science; modelling; linguistics and management. Cultural complexity was therefore considered from diverse perspectives (Figure 2).

\section{Panel discussion and emergent themes}

Dr Anne Kandler, of the Max Planck Institute for Evolutionary Anthropology, chaired our panel discussion. Fittingly, University of Stirling panel member, Prof Christine Caldwell, shared the stage with her former PhD supervisor, Prof Andrew Whiten (University of St Andrews), and former PhD student, Dr Claire Watson (Kyoto University). Dr Nathan Emery (Queen Mary University London), Dr Nicolas Claidière (Aix-Marseille University), and Dr Claudio Tennie (University of Tübingen) also joined the fray. Together our panel represented scientists from experimental psychology, animal behaviour, comparative cognition, and archaeology, and our audience added more complexity still. Kandler presented the panel with three main topics for discussion (see sections in bold), which encompassed themes highlighted throughout the aforementioned talks and posters. 
When asked to provide an explanation as to how complexity is used in the examination of culture within their discipline, panel members came to no consensus. Rather, some researchers stated that they focus on the complexity of the product, and others the complexity of the process whereby the cultural product is created. For example, in archaeology the complexity of a product is often represented by the number of technounits it contains or by an inferred minimum number of intermediary steps, but as Tennie stated, archaeologists do not have the luxury of being able to directly examine the processes involved in product production. Conversely, Emery, from the field of comparative cognition, specified that when he considers complexity he is generally thinking about complex cognition. That is, higher-representational levels and how individual units build upon/interact with each other, rather than perceptual responses to stimuli. Whiten described how in his research, better representing the complexities of the real world has been a major goal. For example, in his recent research exposing children and chimpanzees to micro-worlds affording cumulative cultural change, complexity is multifaceted because tools, actions, and even the decision process needed for task success, exhibit rising levels of complexity. ${ }^{1}$ Even researchers within the same field (animal behaviour) differed in whether they considered complexity in terms of the process or the product, highlighting the divisive nature of this widely used, yet poorly defined, term. Caldwell (previously inspired by audience member, Dr Mark Atkinson, University of Stirling) made the suggestion that the term cumulative cultural evolution (CCE) could be reserved for describing the process which produces accumulated products, and the products themselves referred to as cumulative culture (CC).

Bandini and Reindl had previously highlighted the issue of complexity in process versus product in their keynote talk, questioning the necessity of increasing complexity as a criterion for cumulative cultural evolution. Showing pictures of a lighter and a match, they asked the audience which came first, before revealing that the lighter came first. This simple yet effective example was used to highlight that the evolution of some cultural traits involve decreases in complexity, suggesting that increases in complexity of the product should not be regarded as a defining feature of CCE. However, it was noted that an increase in efficiency of product use may require an increasingly complex production process, which confuses matters somewhat.

How do these different researchers measure the aspects of complexity most relevant to them? And, when considering cumulative cultural evolution, are we more interested in relative measures, rather than absolute magnitude? If complexity within cultural evolutionary research is considered differently by different disciplines, is it a concept which can be consistently operationalised?

It seems there is no simple answer to these questions surrounding the measurement of complexity, especially when comparing across disciplines, described by Kandler as the "gold star challenge" in this field. Emery pointed out that the measurement of complexity appears 
to be domain specific, and that attempting to "simplify complexity" into a measure that can be used across disciplines would surely be detrimental to the field. In the same vein, audience member Prof Christian Rutz (University of St Andrews) described comparisons across taxa as the "real challenge" and highlighted the difficulty presented by natural constraints which differ between species. He therefore suggested that researchers in different disciplines choose the metric which best allows them to measure directional change in their chosen system before comparisons across systems are made. Whiten showed, however, that all is not lost and useful comparisons can be made across different species when he referenced work by Claidière, who is using algorithmic measures of complexity similar to those used in linguistics research. Linguistic research was also brought into the discussion by Caldwell in reference to the clearly-defined metrics used. These have nonetheless failed to resolve debates between linguists as to "what is a complex thing." Caldwell suggested that as a field it would be helpful if we could agree on a broad standard which, as a minimum, allows us to state that one thing is more complex than another, allowing for greater communication between disciplines even if the way complexity is measured differs between them. How easy this will be, is yet to be determined.... As Watson commented, there are many different aspects of complexity to consider. In addition to the complexity of the process and the product, we must also consider the cognitive demands imposed by different tasks.

Prior to the discussion, the panel members and audience alike voted on the question below using an interactive system.

A defining feature of cumulative cultural evolution is often cited as an increase in the complexity of traits/behaviours across generations, but does the term complex fully encapsulate the changes we want to describe? Some authors have identified additional means whereby a trait/behaviour can change over generations to become more useful, therefore should we be describing the changes to traits/behaviours as an increase in:

- Complexity only

- Complexity and/or efficiency

- Functionality - as an umbrella term incorporating complexity, efficiency and others e.g. security, convenience

- Robustness

- None of the above

The overwhelming majority of delegates voted for 'functionality', with about $10 \%$ selecting 'complexity and/or efficiency', and a few brave individuals choosing 'none of the above'. This vote highlighted that many academics are dissatisfied with using complexity to describe modified traits/the process during which traits are modified in the context of cumulative cultural evolution, and therefore the need for further discussion. Issues with using the term complexity were emphasised earlier in the conference by Bandini and Reindl in their keynote talk - 'Complexity: an inefficient concept for the study of culture'. They presented three problems: inconsistencies in assessing the degree of complexity for the same trait across different domains (for example: artifacts, knowledge, behaviour, cognitive 
processes), the subjectivity of judgments made about levels even within these domains, and lastly, that increasing complexity is not an inevitable outcome of CCE. Building on this last point, Bandini and Reindl shared examples in which the evolution of cultural traits shows a decrease in complexity, and explained why using the criterion of efficiency may be more appropriate.

The opportunity for further discussion into complexity and CCE was provided following Kandler's final panel question: Is complexity enough to describe/define cumulative cultural evolution? This question brought two issues to the forefront: - first, that the concept of complexity may not adequately describe what happens to traits during the ratcheting process in all instances of CCE (for which there are many, varied examples), and secondly, that complexity may thus not be a suitable candidate for one of the core criteria of CCE.

The question immediately sparked comments as to the criteria we use when defining CCE. Whiten started the dialogue, voicing his opinion that complexity need not be thought of as one of the essential criteria when examining potential instances of CCE; rather, very basic or core criteria like those of Mesoudi \& Thornton ${ }^{2}$ - repeated build-up of socially-learned traits - can provide a definition of CCE, then how other features, such as complexity, may or may not emerge, can be further studied. In a supporting point (and in line with thoughts presented by Bandini and Reindl), Rutz reminded us that when talking about the accumulated products of CCE we are often referring to something which has undergone a decrease in complexity together with a paralleled increase in efficiency. Kandler related this point to the idea of robustness, stating that a trait may need to decrease in complexity in order to remain robust, that is to remain within a population and be transmitted between individuals. Once the trait has been adopted it could then increase in complexity once again, expressing an emergent balance between robustness and complexity.

Noting that the panel members were relatively satisfied with labelling a cultural product/process as CCE without necessarily observing increases in complexity, Caldwell posed the questions: "Can we envisage a situation where complexity might increase but we might say that's not cumulative culture? Is that definitely cumulative culture if complexity is increased, or are there circumstances in which that could happen and we could say - no it's not?"

On this point, the panel seemed somewhat divided. For example, Watson (who has studied behavioural traditions in non-human primates extensively) stated that she would not consider non-functional increases in the complexity of behaviours (such as stone handling in Japanese Macaques ${ }^{3}$ ) across generations to represent examples of CCE, and that in her opinion "there has to be some ratcheting, some stepped improvement, rather than simply diversification." Conversely, Whiten said that he would include examples such as these within the domain of CCE (if the behaviours diversify and are then spread via social learning), but that this would just represent one form of CCE, whereas in another form "each element may become more complex, like a particular type of tool use."

Kandler moved the discussion on, leading audience member, Atkinson, to assert that he had voted 'none of the above' in response to the earlier interactive question. In his opinion, we have an intuitive understanding of the word complexity, which can lead to it being 
inadequately defined for the particular context in which it is being used. Describing changes in 'functionality' may instead be better, as the 'function' of a trait is more obviously dependent on the context and type of trait, and therefore we may be more likely to define exactly what we mean by 'function' for any given context. The idea of more precisely defining changes in the element of culture we are addressing, as appropriate to each specific research question, suggested earlier in the discussion by Emery appeared to be met by general approval.

The overall consensus of the panel, and indeed the majority of audience members, seemed to be that complexity does not necessarily need to be present in our definition of CCE. One possible solution to the misinterpretation and ambiguity of particular 'buzz words' used in the literature, is to move towards more explicitly defining the cultural changes we measure in a particular context, whether we use complexity, efficiency, functionality, robustness, a combination of these, or any other term or terms. Atkinson and Caldwell made the important point that, should we move towards using 'functionality', we need to ensure our definitions are clear, or alternatively we might require a 'Functionality in Culture' themedconference next year! Culture Conference 2019 will be held at the University of Stirling, but the exact theme is yet to be decided upon. We are, however, excited to announce that Prof Cecilia Heyes has given informal acceptance to our invitation to give a keynote presentation.

Acknowledgments: We expressly thank our funders, the European Research Council (ERC), and conference organisers Donna Kean, Charlotte Wilks, Dr Mark Atkinson, Kirsten Blakey, Juliet Dunstone, Gemma MacKintosh, Dr Elizabeth Renner and Prof Christine Caldwell. We again thank Prof Christine Caldwell, in addition to Dr Mark Atkinson, Dr Nicolas Claidière, Dr Anne Kandler, Dr Claudio Tennie, Dr Claire Watson and Prof Andrew Whiten, for feedback on the article and all speakers/panel members for their presentations and insights into 'complexity in culture.'

Culture Conference 2018: "Complexity in Culture" received funding from the European Research Council (ERC) under the European Union's Horizon 2020 research and innovation programme. Grant agreement No. 648841 RATCHETCOG ERC-2014-CoG.

\section{References}

[1] McGuigan, N., Burdett, E., Burgess, V., Dean, L., Lucas, A., Vale, G., \& Whiten, A. (2017). Innovation and social transmission in experimental micro-societies: exploring the scope of cumulative culture in young children. Phil. Trans. R. Soc. B. 372, 20160425.

[2] Mesoudi, A., \& Thornton, A. (2018). What is cumulative cultural evolution? Proc. R. Soc. B. 285, 20180712.

[3] Schofield, D.P., McGrew, W.C., Takahashi, A., \& Hirata, S. (2018). Cumulative culture in nonhumans: overlooked findings from Japanese Monkeys? Primates, 59, 113-122. 
Figures

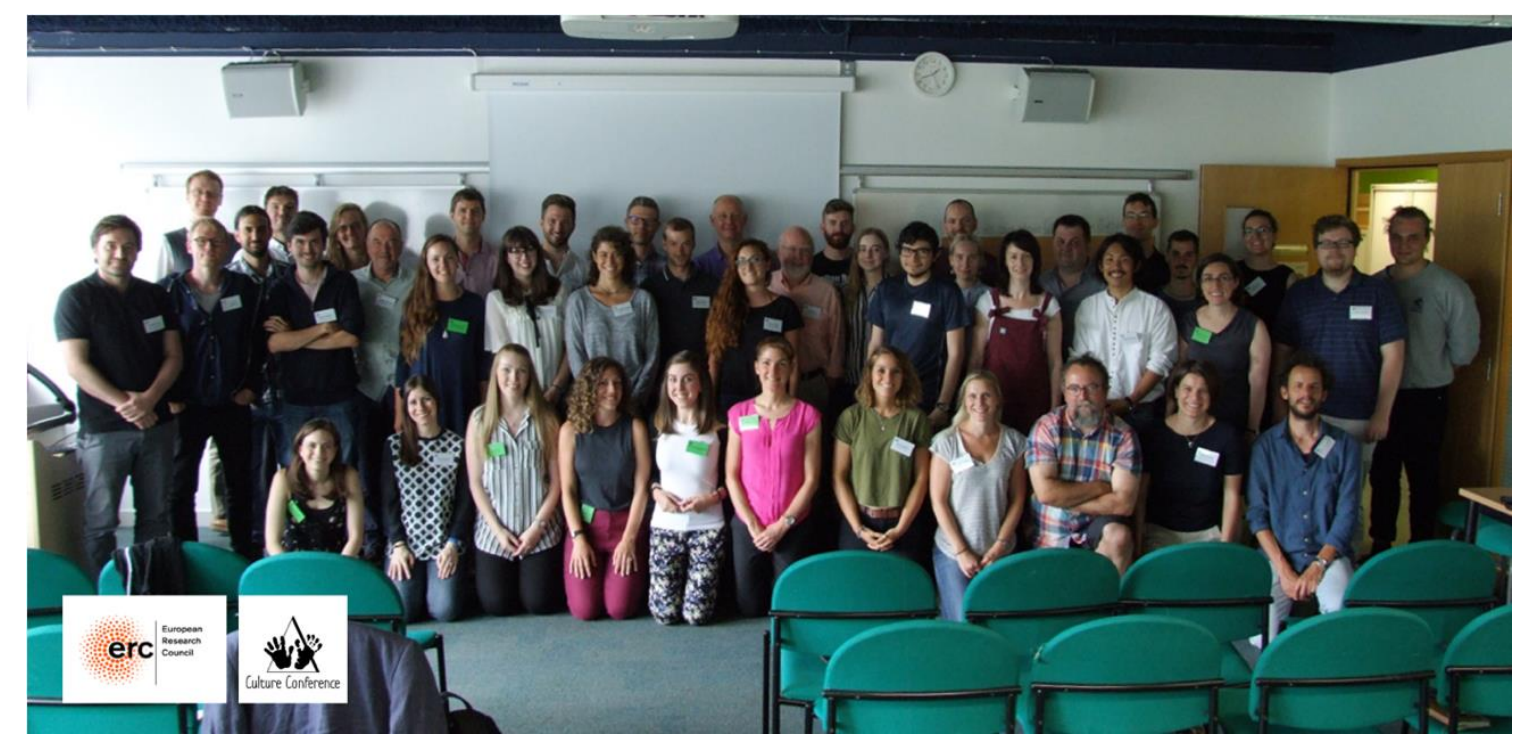

Fig 1: The attendees of the Culture Conference 2018: "Complexity in Culture".

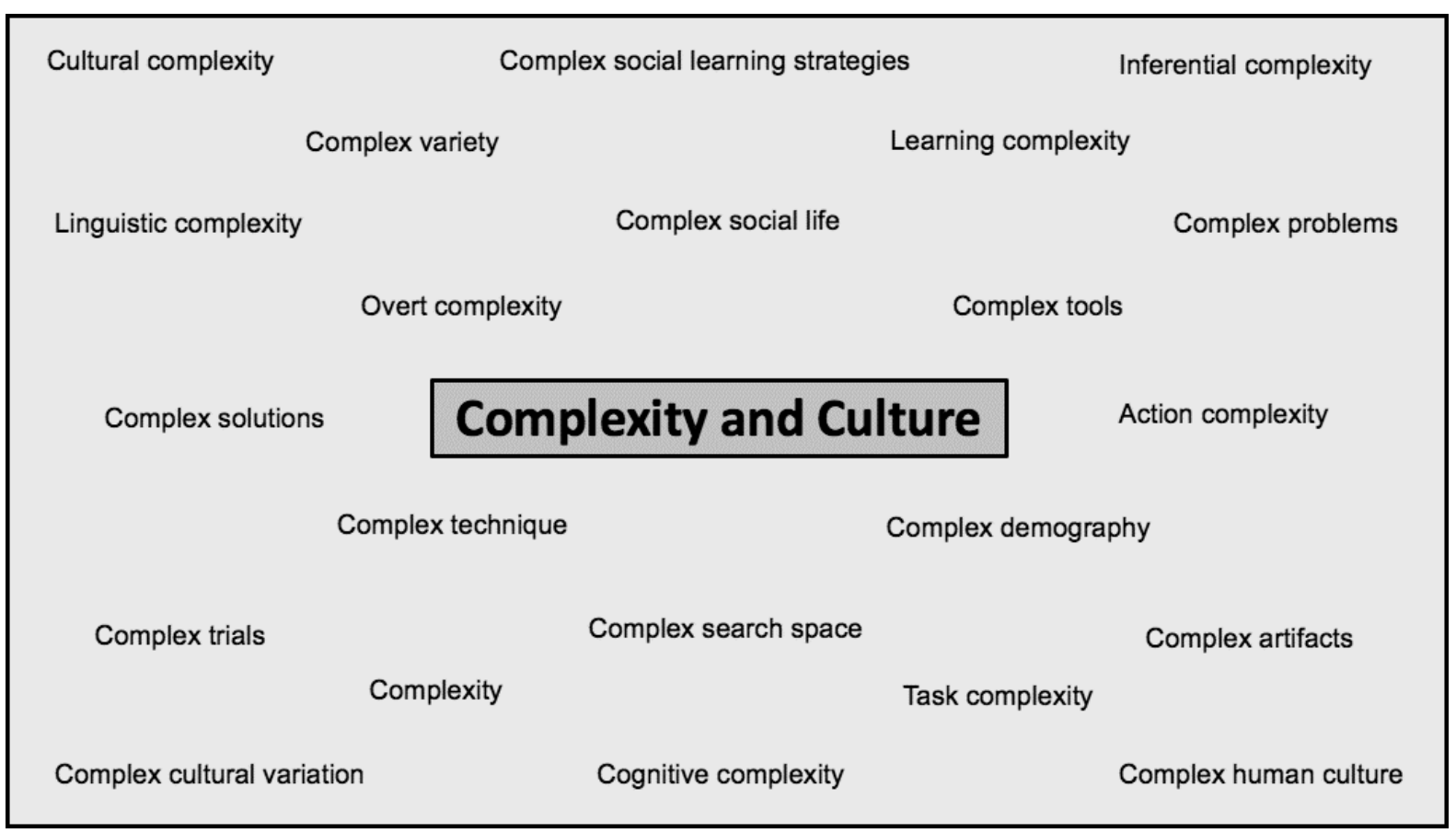

Fig 2: A depiction of the use of the words complex/complexity from poster and talk abstracts. 
